\title{
Special issue of the ISMP 2012 in Berlin
}

\author{
Michael Hintermüller • Martin Skutella
}

Published online: 8 July 2012

(C) Springer and Mathematical Optimization Society 2012

This special issue is related to the 21st International Symposium on Mathematical Programming (ISMP) which took place in Berlin (Germany) from August 19-24, 2012 , with probably the largest attendance in the history of meetings organized by the Mathematical Optimization Society. Like the previous symposia, the 21st ISMP covered a broad range of topics in mathematical optimization with 15 plenary and semi-plenary speakers highlighting current focus areas in the field. This special issue of Mathematical Programming, Series B offers a compilation of papers motivated by contributions of the keynote speakers. The covered areas range from topics in combinatorial, semidefinite, and nonlinear programming to applications in economics, environmental sciences related to electricity markets, molecular sciences and many more.

The contribution by N. Bansal surveys some recent applications of semidefinite optimization in connection with discrepancy theory. The discrepancy of a set system is related to a red-blue coloring of the ground set such that each set in the system is colored as evenly as possible. This concept has close connection to the question of rounding a non-integral solution of a linear system to an integer solution.

D. Bertsimas et al. introduce a method that uses ideas and techniques from robust optimization for stochastic analysis to get computational tractability. This new approach is applied to important applications in different areas.

Smoothing methods for minimization problems where the feasible set is convex but the objective function is not convex, not differentiable and perhaps not even locally Lipschitz at the solutions are the focus of the work contributed by X. Chen. Such

\footnotetext{
M. Hintermüller $(\varangle)$

Humboldt-Universität zu Berlin, Berlin, Germany

e-mail: hint@math.hu-berlin.de

M. Skutella

Technische Universität Berlin, Berlin, Germany
} 
challenging non-Lipschitz optimization problems arise from wide applications including image restoration, signal reconstruction, variable selection, optimal control, stochastic equilibrium and spherical approximations.

Extremely large scale, but structured minimization problems arise in inversion problems including differential equations modeling the underlying physical system, but they arise also in problems, for instance, related to speech recognition. Due the tremendous size of the problem instances, reduction techniques are required. In his contribution, M. Friedlander et al. survey several dimensionality-reduction techniques based on data averaging or sampling and study their connection to stochastic optimization. It turns out that data-averaging approaches are only viable for a least-squares misfit, which is sensitive to outliers in the data and artifacts unexplained by the forward model. As a remedy a robust formulation based on the Student's t-distribution of the error is proposed.

Still in view of the previously mentioned problem classes (highlighting the particular application of speech recognition) J. Nocedal et al. present a methodology for using varying sample sizes in batch-type optimization methods for large-scale machine learning problems. For a variance based sample size increase they establish an $\mathcal{O}(1 / \epsilon)$ complexity bound on the total cost of a gradient method. Among other interesting aspects, the paper covers minimization problems involving an $L^{1}$-regularization in order to promote sparse solutions.

Convexity arises naturally in financial risk management. For instance, in risk preferences concerning random cash-flows, convexity corresponds to the fundamental diversification principle. Convexity is a basic property also of budget constraints both in classical linear models as well as in more realistic models with transaction costs and constraints. Moreover, modern securities markets are based on trading protocols that result in convex trading costs. In his contribution, T. Pennanen provides an introduction to convex optimization in financial markets and covers a broad range of analytical as well as numerical issues.

"Divide to conquer", as a motive for the derivation of decomposition methods for energy optimization, is the focus of C. Sagastizábal's paper. The increasing penetration of low carbon renewable sources of energy introduces uncertainty in electricity systems traditionally modeled in a deterministic setting. The liberalization of the electricity sector brought the need of designing sound markets, ensuring capacity investments while properly reflecting strategic interactions. In all these problems, hedging risk, possibly in a dynamic manner, is also a concern. Besides a wide range of adequate models, C. Sagastizábal explores some variants of solution methods based on Lagrangian relaxation and on Benders decomposition.

K. Scheinberg et al. study sparse low degree interpolating polynomials and their application to derivative-free optimization. Often, in practical applications, the contribution of the problem variables to the objective function is such that many pairwise correlations between variables are negligible, implying, in the smooth case, a sparse structure in the Hessian matrix. To be able to exploit Hessian sparsity, existing optimization approaches require the knowledge of the sparsity structure. For the latter, sparse recovery theory developed recently in the field of compressed sensing turns out to be useful in the setting of the contribution of K. Scheinberg et al. 
In the paper contributed by C. Schütte et al. the optimal control of a molecular conformational ensemble in an infinite time horizon is addressed. The high dimensional Hamilton Jacobi Bellman equation is interpreted as a nonlinear eigenvalue problem. The authors propose and show a new way, how this equation can be transformed to a linear eigenvalue problem which can then be approximated in a Markov state model setting.

Mechanism design is an analytical paradigm that has been influential in modern economics. The paper by R.V. Vohra provides an overview on classical and recent work in optimal mechanism design and about the role that mathematical programming techniques can play. In particular it is shown how polymatroid optimization shows up.

R. Weismantel et al. address the minimization of Lipschitz-continuous and strongly convex functions over integer points in polytopes. The authors present complexity results based on an iterative algorithm that solves a sequence of special quadratic integer problems.

Finally, after this impressive list of contributions to this special issue, the editors would like to thank the keynote speakers for preparing their manuscripts in time such that the editors' promise to have the special issue available already at the time of the symposium could be kept. Of course, this also requires very disciplined reviewers, whom we also would like to thank very much. Last but not least, we would like to express our sincere gratitude to the editor-in-chief of Mathematical Programming B, Danny Ralph, who was always swift in replies to our requests and supportive in all respects, and to the Springer production staff for their flexibility and support in the overall production process.

At the end, we would like to thank the scientific committee for their support and the local organizing committee for all of their efforts which made the symposium an outstanding success. 